Pacific Journal of Mathematics

RIEMANN SURFACES WHICH ARE DOUBLES OF PLANE 


\title{
RIEMANN SURFACES WHICH ARE DOUBLES OF PLANE DOMAINS
}

\author{
N. S. HAWLeY AND M. M. SChiffer
}

\begin{abstract}
In our investigations of Riemann surfaces we have had occasion to need a criterion which will distinguish from among all closed Riemann surfaces those which are doubles of plane domains. We give here a criterion which we have found useful in practice.
\end{abstract}

The technique of doubling a plane domain, as a tool in conformal mapping, was introduced by Schottky in 1875 and used by him, by Picard, by Klein and by many others since [3]. In particular this idea led Klein to introduce the symmetric Riemann surfaces.

Let us denote by $\boldsymbol{P}_{n}(\boldsymbol{C})$ the complex projective $n$ space. A point $\zeta$ of $\boldsymbol{P}_{n}(\boldsymbol{C})$ is given by the $(n+1)$ homogeneous coordinates $\left(\zeta_{1}, \cdots, \zeta_{n+1}\right)$. Let $\boldsymbol{P}_{n}(\boldsymbol{R})$ denote the real projective $n$ space, which we consider imbedded in $\boldsymbol{P}_{n}(\boldsymbol{C})$ : it consists of those points $\left(\zeta_{1}, \cdots, \zeta_{n+1}\right)$ such that the ratios of the homogeneous coordinates are real.

Consider a compact Riemann surface $\mathscr{R}$ of genus $p$, and let $\omega_{1}, \cdots, \omega_{p}$ be a basis of differentials of the first kind on $\mathscr{R}$. Any such basis defines an analytic mapping

$$
\text { ע: } \mathscr{R} \rightarrow \boldsymbol{P}_{p-1}(\boldsymbol{C})
$$

obtained explicitly as follows: for $\mathfrak{q} \in \mathscr{R}$,

$$
\boldsymbol{\nu}(\mathfrak{q})=\left(\omega_{1}(\mathfrak{q}), \cdots, \omega_{p}(\mathfrak{q})\right) \in \boldsymbol{P}_{p-1}(\boldsymbol{C}) .
$$

This mapping, known as the Noether mapping [1], is an analytic homeomorphism of $\mathscr{R}$ into $\boldsymbol{P}_{p-1}(\boldsymbol{C})$ if $p>2$ and $\mathscr{R}$ is not hyperelliptic. (The mapping appears to depend on the basis $\omega_{1}, \cdots, \omega_{p}$ of differentials of the first kind chosen on $\mathscr{R}$; but if to any change of the basis $\omega_{1}, \cdots, \omega_{p}$ we introduce the cogredient change of coordinates in $\boldsymbol{P}_{p-1}(\boldsymbol{C})$, the mapping is even unique!)

Now let $\mathscr{D}$ be a $(p+1)$-ply connected plane domain bounded by $p+1$ analytic Jordan curves $C_{0}, C_{1}, \cdots, C_{p}$, and let $\mathscr{R}$ be its double [3]. Then $\mathscr{R}$ is a compact Riemann surface of genus $p$. Let $\omega_{1}, \cdots, \omega_{p}$ be a basis of Schottky (or "real") differentials on $\mathscr{D}$. This basis has a natural (and unique) extension to a basis of differentials of the first kind on $\mathscr{R}$, and thereby defines an analytic mapping of $\mathscr{R}$ (and therefore $\mathscr{D})$ into $\boldsymbol{P}_{p-1}(\boldsymbol{C})$. Since the ratios of the $\omega_{j}$ are real on the boundary curves $C_{k}$, the images $\mathscr{C}_{k}$ of these curves under $\boldsymbol{\nu}$ lie in $\boldsymbol{P}_{p-1}(\boldsymbol{R})$, the real projective space. If $\mathscr{R}$ is nonhyperelliptic, the $\mathscr{C}_{k}$ are mutually disjoint, since $\nu$ is a homeomorphism. 
That $\mathscr{R}$ intersects $\boldsymbol{P}_{p-1}(\boldsymbol{C})$ in this manner discloses an interesting property of Riemann surfaces which are the doubles of plane domains. It is a property not shared by all closed Riemann surfaces, for some of them have images under the Noether map which do not even intersect $\boldsymbol{P}_{p-1}(\boldsymbol{R})$ at all. In fact, we are going to show that this intersection property is almost completely characteristic of those Riemann surfaces which are the doubles of plane domains.

In order to state the assertion precisely, we make the following definition:

DeFinition 1. The compact Riemann surface $\mathscr{R}$ of genus $p$ is said to have the property $\Pi_{1}$ if $p>2$ and there exists a basis $\omega_{1}, \cdots, \omega_{p}$ of differentials of the first kind on $\mathscr{R}$ such that the image $\mathscr{R}_{\nu}$ of $\mathscr{R}$, under the Noether map $\boldsymbol{\nu}$ defined by this basis, intersects $\boldsymbol{P}_{p-1}(\boldsymbol{R})$ in $p+1$ mutually disjoint Jordan curves.

We can now introduce our assertion as a theorem.

THEOREM 1. The Riemann surface $\mathscr{R}$ is nonhyperelliptic and the double of a plane domain if and only if it has the property $\Pi_{1}$.

In order to prove Theorem 1 we first prove several lemmas which are of some interest in themselves and which, in fact, contain information beyond that necessary to prove Theorem 1 .

On each $\mathbb{P}_{n}(\boldsymbol{C})$ there is a natural antianalytic involution

$$
*: \boldsymbol{P}_{n}(\boldsymbol{C}) \rightarrow \boldsymbol{P}_{n}(\boldsymbol{C})
$$

defined by

$$
* \zeta=\left(\bar{\zeta}_{1}, \cdots, \bar{\zeta}_{n+1}\right), \text { where } \zeta=\left(\zeta_{1}, \cdots, \zeta_{n+1}\right) .
$$

If $\mathscr{R} \subset \boldsymbol{P}_{n}(\boldsymbol{C})$ is a Riemann surface which is a complex analytic submanifold of $\boldsymbol{P}_{n}(\boldsymbol{C})$, and if we denote its image under the mapping $*$ by $* \mathscr{R}$, then $* \mathscr{R}$ is an antianalytic submanifold of $\boldsymbol{P}_{n}(\boldsymbol{C})$. In any case, we can consider $\boldsymbol{P}_{n}(\boldsymbol{C})$ as a $2 n$-dimensional real analytic manifold and with respect to this real structure both $\mathscr{R}$ and $* \mathscr{R}$ are real analytic submanifolds of $\boldsymbol{P}_{n}(\boldsymbol{C})$.

As real analytic submanifolds of an analytic manifold, if $\mathscr{R}$ and * $\mathscr{R}$ coincide in a neighborhood of any common point, they must coincide globally. This follows from the uniqueness of analytic continuation; for if $\mathscr{R}$ and $* \mathscr{R}$ coincide in a neighborhood of some point, there is a neighborhood of that point in which both submanifolds can be defined (locally) by the same analytic equations. But each manifold is defined globally by analytic continuations of these equations.

We can now make the following assertion: 
Lemma 1. If $\mathscr{R}$ is a compact Riemann surface which is an analytic submanifold of $\boldsymbol{P}_{n}(\boldsymbol{C})$, and $\mathscr{C}$ is an analytic arc which is contained in the intersection of $\mathscr{R}_{\nu}$ with $\boldsymbol{P}_{n}(\boldsymbol{R})$, then $* \mathscr{R}=\mathscr{R}$.

Proof. Since $\boldsymbol{P}_{n}(\boldsymbol{R})$ remains pointwise fixed under $*$, the hypotheses of the lemma imply that $\mathscr{R}$ must contain an analytic arc $\mathscr{C}$ which remains pointwise fixed under $*$. Let $\mathfrak{q}_{0} \in \mathscr{C}$ and $z$ be a local uniformizing parameter defined in a neighborhood $U_{0}$ of $\mathfrak{q}_{0}$ so that $z: U_{0} \rightarrow \boldsymbol{C}$, $z\left(\mathfrak{q}_{0}\right)=0$ and $z$ maps $\mathscr{C} \cap U_{0}$ into the real axis in $\boldsymbol{C}$. (We can choose $U_{0}$ small enough so that this is possible.) Next, choose $\varepsilon$ so small that the disc $E_{\varepsilon}=\{z:|z|<\varepsilon\}$ is mapped into $U_{0}$ by $z^{-1}$ (note: $z^{-1}$ denotes the inverse mapping of the uniformizer $z$, not the quantity $1 / z)$. Let $U_{0}^{\prime}$ denote its image, i.e., $U_{0}^{\prime}=z^{-1}\left(E_{\varepsilon}\right)$. As $\mathfrak{q} \in \mathscr{R}$ ranges over $U_{0}^{\prime}$, its coordinates $\left(\zeta_{1}, \cdots, \zeta_{n+1}\right)$ in $\boldsymbol{P}_{n}(\boldsymbol{C})$ are given by analytic functions $\varphi_{j}(z(q))$, i.e., $\zeta_{j}=\varphi_{j}(z)$ describes the (inclusion) mapping of $U_{0}^{\prime}$ into $\boldsymbol{P}_{n}(\boldsymbol{C})$. Since $\varphi_{j}(z)$ is analytic and real for $z$ real, we have

$$
\varphi_{j}(\bar{z})=\overline{\varphi_{j}(z)} \text { for }|z|<\varepsilon .
$$

Therefore $*$ maps $U_{0}^{\prime}$ onto itself. But this means that $U_{0}^{\prime}$ is also a neighborhood on $* \mathscr{R}$, therefore $* \mathscr{R}$ must coincide with $\mathscr{R}$ (as a subset of $\left.\boldsymbol{P}_{n}(\boldsymbol{C})\right)$. This proves the lemma.

Let $\mathscr{R}$ be a surface (not necessarily compact, analytic, or even orientable) and let $C_{0}, \cdots, C_{l}$ be a collection of mutually disjoint Jordan curves on $\mathscr{R}$ such that the entire collection separates $\mathscr{R}$, but no proper subcollection of the curves has this property. We call such a collection of Jordan curves a minimal separating set of Jordan curves on $\mathscr{R}$. We can now make the following assertion.

Lemma 2. If $C_{0}, \cdots, C_{l}$ is a minimal separating set of Jordan curves on $\mathscr{R}$, then it separates $\mathscr{R}$ into exactly two connected components.

$$
\begin{aligned}
& \text { Proof. Let } \mathscr{S}_{l}=\mathscr{R}-\bigcup_{k=0}^{l} C_{k} \text { and let } \\
& \qquad \mathscr{S}_{l-1}=\mathscr{R}-\bigcup_{k=0}^{l-1} C_{k} .
\end{aligned}
$$

Then by hypothesis $\mathscr{S}_{l-1}$ is connected and

$$
\mathscr{S}_{l}=\mathscr{S}_{l-1}-C_{l} \text {. }
$$

Suppose that $\mathscr{S}_{l}$ has at least three connected components; we may write

$$
\mathscr{S}_{l}=\mathscr{S}^{\prime} \cup \mathscr{S}^{\prime \prime} \cup \mathscr{S}^{\prime \prime \prime}
$$

where $\mathscr{S}^{\prime}, \mathscr{S}^{\prime \prime}$ and $\mathscr{S}^{\prime \prime \prime}$ are all nonempty and disjoint. Since $\mathscr{R}$ is 
a surface, $C_{l}$ is in the boundary (in $\mathscr{R}$ ) of at most two of the sets $\mathscr{S}^{\prime}, \mathscr{S}^{\prime \prime}, \mathscr{S}^{\prime \prime \prime}$; say, for example, it does not lie in the boundary of $\mathscr{S}^{\prime \prime \prime}$. By assumption $\mathscr{S}^{\prime \prime \prime}$ contains at least one component of $\mathscr{S}_{l}=$ $\mathscr{S}_{l-1}-C_{l}$, but not all of $\mathscr{S}_{l}$. Since $C_{l}$ is disjoint, even from the closure of $\mathscr{S}^{\prime \prime \prime}, \mathscr{S}^{\prime \prime \prime}$ also contains at least one component of $\mathscr{S}_{l-1}$. But this contradicts the assumption that $\mathscr{S}_{l-1}$ is connected. Therefore $\mathscr{S}_{l}$ must consist of at most two components. Since $\mathscr{S}_{l}$ is not connected, it must have exactly two components. This proves the lemma.

We next prove the following lemma.

Lemma 3. Let $\mathscr{R}^{\prime} \subset \boldsymbol{P}_{n}(\boldsymbol{C})$ intersect $\boldsymbol{P}_{n}(\boldsymbol{R})$ in $(m+1)$ mutually disjoint Jordan curves $\mathscr{C}_{0}, \cdots, \mathscr{C}_{m}$, which we suppose separate . $\mathscr{R}^{\prime}$. Then no proper subcollection of these curves separates $\mathscr{R}^{\prime}$.

Proof. Let $l \leqq m$ be the smallest number such that there are $l+1$ curves, say $\mathscr{C}_{0}, \cdots, \mathscr{C}_{l}$ which separate $\mathscr{R}^{\prime}$. Then by Lemma 2

$$
\mathscr{R}^{\prime}-\bigcup_{k=0}^{l} \mathscr{C}_{k}=\mathscr{S}^{\prime} \cup \mathscr{S}^{\prime \prime}
$$

where $\mathscr{S}^{\prime}$ and $\mathscr{S}^{\prime \prime}$ are disjoint nonempty connected components of $\mathscr{R}^{\prime}-\bigcup_{k=0}^{l} \mathscr{C}_{k}$. Now suppose that $l<m$. Let $\mathfrak{q}_{0} \in \mathscr{C}_{0}$ and let $U_{0}$ be a neighborhood of $q_{0}$. Since $\mathscr{C}_{0}$ is in the separating set in $\mathscr{R}^{\prime}, U_{0}$ contains points in both $\mathscr{S}^{\prime}$ and $\mathscr{S}^{\prime \prime}$. As we have seen, in the proof of Lemma 1 , we can choose $U_{0}$ so that $* U_{0}=U_{0}$. Then we can choose $\mathfrak{q}_{1} \in U_{0}$ so that $\mathfrak{q}_{1} \in \mathscr{S}^{\prime}$ and $* \mathfrak{q}_{1} \in \mathscr{S}^{\prime \prime}$. By Lemma $1, *\left(\mathscr{S}^{\prime} \cup \mathscr{S}^{\prime \prime}\right)=$ $\mathscr{S}^{\prime} \cup \mathscr{S}^{\prime \prime}$. Since $*: \mathfrak{q}_{1} \rightarrow * q_{1}$ and $\mathscr{S}^{\prime}$ is connected, $* \mathscr{S}^{\prime} \subset \mathscr{S}^{\prime \prime}$. Likewise $* \mathscr{S}^{\prime \prime} \subset \mathscr{S}^{\prime}$. Therefore (since $\left.*\left(\mathscr{S}^{\prime} \cup \mathscr{S}^{\prime \prime}\right)=\mathscr{S}^{\prime} \cup \mathscr{S}^{\prime \prime}\right)$ we have $* \mathscr{S}^{\prime}=$ $\mathscr{S}^{\prime \prime}$ and $* \mathscr{S}^{\prime \prime}=\mathscr{S}^{\prime}$. Since we assumed $l<m, \mathscr{C}_{m}$ must be contained either in $\mathscr{S}^{\prime}$ or $\mathscr{S}^{\prime \prime}$, say $\mathscr{S}^{\prime}$. But $C_{m}$ remains pointwise fixed under *. Therefore $C_{m} \subset * \mathscr{S}^{\prime}=\mathscr{S}^{\prime \prime}$, which contradicts the assumption that $\mathscr{S}^{\prime}$ and $\mathscr{S}^{\prime \prime}$ are disjoint. Therefore $l=m$ and the lemma is proved. We are now in a position to prove Theorem 1.

Proof of Theorem 1. First we note that if $\mathscr{R}$ is hyperelliptic, then $\mathscr{R}_{2}$ is of genus zero so that any Jordan curve on $\mathscr{R}_{y}$ separates it. Therefore, by Lemma 3, $\mathscr{R}_{\nu}$ cannot meet $\boldsymbol{P}_{p-1}(\boldsymbol{C})$ in $p+1$ Jordan curves. Hence if $\mathscr{R}$ has property $\Pi_{1}$, then it is not hyperelliptic. Next we note that since $\mathscr{R}_{\nu}$ is a compact orientable surface of genus $p$, any $p+1$ mutually disjoint Jordan curves separate it. By Lemma 2 $\mathscr{R}_{\nu}-\bigcup_{k=0}^{p} \mathscr{C}_{k}=\mathscr{S}^{\prime} \cup \mathscr{S}^{\prime \prime}$, where $\mathscr{S}^{\prime}$ and $\mathscr{S}^{\prime \prime}$ are connected, disjoint from one another and $* \mathscr{S}^{\prime}=\mathscr{S}^{\prime \prime}$ and $* \mathscr{S}^{\prime \prime}=\mathscr{S}^{\prime}$. We can see that $\mathscr{S}^{\prime}$ is homeomorphic to a plane domain as follows: since $\mathscr{R}_{\nu}$ is homeomorphic to a sphere with $p$ handles, we can choose the handles so that $\mathscr{C}_{1}, \cdots, \mathscr{C}_{p}$ lie on separate handles. Removal of $\mathscr{C}_{1}, \cdots, \mathscr{C}_{p}$ 
from $\mathscr{R}_{\nu}$ leaves us with a surface which is homeomorphic to a sphere with $2 p$ holes cut in it. Removal of $\mathscr{C}_{0}$ from this residual surface leaves us with exactly two pieces (which are homeomorphic to one another under $*$ ). Each of these pieces is topologically equivalent to a plane domain bounded by $p+1$ Jordan curves. We could now appeal to the uniformization theorem of Koebe [2, p. 474], but we can also sketch an argument here to complete the proof. Let $d t\left(q ; \mathfrak{q}_{0}\right)$ denote a simple Abelian differential of the second kind on $\mathscr{R}$; it has a single pole of order two at $\mathfrak{q}_{0}$ and is regular otherwise. We may also choose $t\left(\mathfrak{q} ; \mathfrak{q}_{0}\right)$ so that its periods around $C_{1}, \cdots, C_{p}$ are all zero. Since $-C_{0}$ is homologous to $C_{1}, \cdots, C_{p}$, the period of $t\left(\mathfrak{q} ; \mathfrak{q}_{0}\right)$ is also zero around $C_{0}$. The antianalytic involution $*$ on $\mathscr{R}_{\nu}$ induces an antianalytic involution, which we also denote by $*$, on $\mathscr{R}$. Clearly, $d t\left(* \mathfrak{q} ; * \mathfrak{q}_{0}\right)=\overline{\operatorname{dt}\left(\mathfrak{q} ; \mathfrak{q}_{0}\right)}$. Now choose $\mathfrak{q}_{0}$ on one of the fixed curves $C_{k}$, say $C_{0}$. Then $* q_{0}=q_{0}$. Therefore if $\mathfrak{q}$ lies on any of the $C_{k}$, we have $* \mathfrak{q}=\mathfrak{q}$ so $d t\left(* \mathfrak{q} ; * \mathfrak{q}_{0}\right)=d t\left(\mathfrak{q} ; \mathfrak{q}_{0}\right)=$ $\overline{d t\left(\mathfrak{q} ; \mathfrak{q}_{0}\right)}$, thus we see that $d t\left(\mathfrak{q} ; \mathfrak{q}_{0}\right)$ is real on each of the $C_{k}$. Then

Let $\mathscr{R}^{+}$denote one of the connected pieces of $\mathscr{R}-\bigcup_{k=0}^{p} C_{k}$.

$$
t\left(\mathfrak{q} ; \mathfrak{q}_{0}\right)=\int d t\left(\mathfrak{q} ; \mathfrak{q}_{0}\right)
$$

is single valued and regular on $\mathscr{R}^{+}$. Also its imaginary part is constant on each $C_{k}$ (and zero on $C_{0}$ ) so we can apply the argument principle to see that $t\left(q ; \mathfrak{q}_{0}\right)$ is univalent in $\mathscr{R}^{+}$. It thus maps $\mathscr{R}^{+}$conformally on to a domain which is the complement of $p+1$ slits which are parallel to the real axis (one of the slits passing through $\infty$ ). This type of domain we know is conformally equivalent to one bounded by $p+1$ Jordan curves. (In fact, even equivalent to one bounded by $p+1$ circles [4].) This completes the proof of the theorem.

We now consider the hyperelliptic case. Suppose the double $\mathscr{R}$ of the plane domain $\mathscr{D}$ is hyperelliptic. The Noether mapping $\nu$ still exists although it is not one-to-one in this case. The image $\mathscr{R}_{2}$ is, in this case, a nonsingular rational curve in $\boldsymbol{P}_{p-1}(\boldsymbol{C})$. However, instead of using linear differentials to define

$$
\nu: \mathscr{R} \rightarrow \boldsymbol{P}_{p-1}(\boldsymbol{C})
$$

we can use quadratic differentials to define

$$
\mu: \mathscr{R} \rightarrow \boldsymbol{P}_{3 p-t}(\boldsymbol{C}) \text {. }
$$

This mapping gives an analytic homeomorphism in all cases where $p>2$, hyperelliptic or not. In the case $p=2$ the image $\mathscr{R}_{\mu}$ is again a rational (plane) curve.

For the case $p=2$ we must take cubic differentials which will then define 


$$
\tau: \mathscr{R} \rightarrow \boldsymbol{P}_{5}(\boldsymbol{C}) .
$$

The case $p=1$ cannot be handled by this method at all; but we can deal with it easily in another (known) manner by using the (one) modulus of the curve. For $p=0$ there is no problem to consider.

Returning now to the mapping $\mu$, we make the following definition.

Definition 2. The compact Riemann surface $\mathscr{R}$ of genus $p$ is said to have the property $\Pi_{2}$ if $p>2$ and there exists a basis $Q_{1}, \cdots, Q_{3 p-3}$ of quadratic differentials of the first kind (i.e., regular) on $\mathscr{R}$ such that the image $\mathscr{R}_{\mu}$ of $\mathscr{R}$ under the mapping $\mu$, defined by this basis, intersects $\boldsymbol{P}_{3 p-i}(\boldsymbol{R})$ in $p+1$ mutually disjoint Jordan curves.

Clearly, the double of a plane domain bounded by $p+1$ analytic Jordan curves (where $p>2$ ) has property $\Pi_{2}$. We can now state a second theorem.

Theorem 2. A closed Riemann surface $\mathscr{R}$ of genus $p>2$ is the double of a plane domain if and only if it has the property $\Pi_{2}$.

The proof of Theorem 2 is exactly like that of Theorem 1. Lemmas 1, 2, and 3 made no use of the dimension of $\boldsymbol{P}_{n}(\boldsymbol{C})$, or of any particular mapping of $\mathscr{R}$ into $\boldsymbol{P}_{n}(\boldsymbol{C})$, they only required that $\mathscr{R}^{\prime}$ (the image of $\mathscr{R}$ ) should be a complex analytic subvariety of $\boldsymbol{P}_{n}(\boldsymbol{C})$.

The case $p=2$ can be dealt with in the same manner, except that we must use cubic differentials. The reason for preferring Theorem 1 over the more inclusive Theorem 2 is that the ordinary differentials of the first kind are entities readily available for use, whereas the quadratic differentials are not so easily accessible-especially in the hyperelliptic case for $p>2$, where there exist quadratic differentials which are not generated from a quadratic form in linear differentials. Thus Theorem 2 would be much harder to apply in actuality in just those cases in which it has any advantage over Theorem 1.

\section{REFERENCES}

1. K. Hensel and G. Landsberg, Theorie der algebraischen funktionen einer variabeln, Leipzig, 1902.

2. A. Hurwitz and R. Courant, Funktionentheorie, Berlin, 1929.

3. M. Schiffer and D. C. Spencer, Functionals of finite riemann surfaces, Princeton, 1954.

4. M. Schiffer and N. S. Hawley, Connections and conformal mapping, Acta Math. 107 (1962), 175-274.

Received March 2, 1966. This work was supported in part by National Science Foundation grant GP 4069 and Air Force contract AF 49 (638) 1345 at Stanford University. 


\section{PACIFIC JOURNAL OF MATHEMATICS}

\section{EDITORS}

\author{
H. SAMELSON \\ Stanford University \\ Stanford, California \\ J. P. JANS \\ University of Washington \\ Seattle, Washington 98105
}

\section{J. DugundJI}

University of Southern California Los Angeles, California 90007

RICHARD ARENS

University of California

Los Angeles, California 90024

\section{ASSOCIATE EDITORS}
E. F. BECKENBACH
B. H. NeumanN
F. WOLF
K. YOSIDA

\section{SUPPORTING INSTITUTIONS}

\author{
UNIVERSITY OF BRITISH COLUMBIA \\ CALIFORNIA INSTITUTE OF TECHNOLOGY \\ UNIVERSITY OF CALIFORNIA \\ MONTANA STATE UNIVERSITY \\ UNIVERSITY OF NEVADA \\ NEW MEXICO STATE UNIVERSITY \\ OREGON STATE UNIVERSITY \\ UNIVERSITY OF OREGON \\ OSAKA UNIVERSITY \\ UNIVERSITY OF SOUTHERN CALIFORNIA
}

\author{
STANFORD UNIVERSITY \\ UNIVERSITY OF TOKYO \\ UNIVERSITY OF UTAH \\ WASHINGTON STATE UNIVERSITY \\ UNIVERSITY OF WASHINGTON \\ AMERICAN MATHEMATICAL SOCIETY \\ CHEVRON RESEARCH CORPORATION \\ TRW SYSTEMS \\ NAVAL ORDNANCE TEST STATION
}

Mathematical papers intended for publication in the Pacific Journal of Mathematics should be typewritten (double spaced). The first paragraph or two must be capable of being used separately as a synopsis of the entire paper. It should not contain references to the bibliography. Manuscripts may be sent to any one of the four editors. All other communications to the editors should be addressed to the managing editor, Richard Arens at the University of California, Los Angeles, California 90024 .

50 reprints per author of each article are furnished free of charge; additional copies may be obtained at cost in multiples of 50 .

The Pacific Journal of Mathematics is published monthly. Effective with Volume 16 the price per volume (3 numbers) is $\$ 8.00$; single issues, $\$ 3.00$. Special price for current issues to individual faculty members of supporting institutions and to individual members of the American Mathematical Society: $\$ 4.00$ per volume; single issues $\$ 1.50$. Back numbers are available.

Subscriptions, orders for back numbers, and changes of address should be sent to Pacific Journal of Mathematics, 103 Highland Boulevard, Berkeley 8, California.

Printed at Kokusai Bunken Insatsusha (International Academic Printing Co., Ltd.), No. 6, 2-chome, Fujimi-cho, Chiyoda-ku, Tokyo, Japan.

PUBLISHED BY PACIFIC JOURNAL OF MATHEMATICS, A NON-PROFIT CORPORATION

The Supporting Institutions listed above contribute to the cost of publication of this Journal, but they are not owners or publishers and have no responsibility for its content or policies. 


\section{Pacific Journal of Mathematics \\ Vol. 20, No. $2 \quad$ October, 1967}

Edward Dewey Davis, Ideals of the principal class, $R$-sequences and a certain monoidal transformation ............................. 197

Richard Mansfield Dudley, Sub-stationary processes ................ 207

Newton Seymour Hawley and M. Schiffer, Riemann surfaces which are doubles of plane domains ......................... 217

Barry E. Johnson, Continuity of transformations which leave invariant certain translation invariant subspaces ................... 223

John Eldon Mack and Donald Glen Johnson, The Dedekind completion of

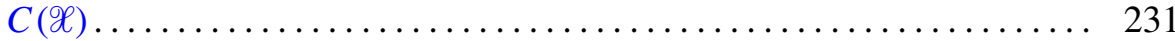

K. K. Mathur and R. B. Saxena, On the convergence of quasi-Hermite-Fejér interpolation................................... 245

H. D. Miller, Generalization of a theorem of Marcinkiewicz............ 261

Joseph Baruch Muskat, Reciprocity and Jacobi sums ............... 275

Stelios A. Negrepontis, On a theorem by Hoffman and Ramsay .......... 281

Paul Adrian Nickel, A note on principal functions and multiply-valent canonical mappings .............................. 283

Robert Charles Thompson, On a class of matrix equations ............. 289

David Morris Topping, Asymptoticity and semimodularity in projection lattices ........................................ 317

James Ramsey Webb, A Hellinger integral representation for bounded linear functionals

Joel John Westman, Locally trivial $C^{r}$ groupoids and their representations...

Hung-Hsi Wu, Holonomy groups of indefinite metrics 